\title{
INSCRIBING SMOOTH KNOTS WITH REGULAR POLYGONS
}

\author{
YING-QING WU ${ }^{1}$
}

\begin{abstract}
A regular $n$-gon inscribing a knot is a sequence of $n$ points on a knot, such that the distances between adjacent points are all the same. It is shown that any smooth knot is inscribed by a regular $n$-gon for any $n$.
\end{abstract}

A knot $K: S^{1} \rightarrow \mathbb{R}^{3}$ is said to be inscribed by a regular $n$-gon if there is a set of points $x_{0}, \ldots, x_{n-1}$ lying on $K$ in a cyclic order, such that the distances $\left\|x_{i-1}-x_{i}\right\|$ between $x_{i-1}$ and $x_{i}$ are the same for $i=1, \ldots, n$, where $x_{n}=x_{0}$. Jon Simon asked the question of whether every smooth knot $K$ is inscribed by a regular $n$-gon for all $n$. There has been quite some research activities on this and related problems. See $[2, \S 11]$ and the references there. In particular, it was shown by Meyerson [4] and E. Kronheimer and P. Kronheimer [3] that given any triangle there is one similar to it which inscribes a given planar curve. It is a very interesting open question whether any closed planar curve is inscribed by a square [2], although this has been proved for a very large class of curves, including all smooth or piecewise linear curves [6]. See also [5].

Up to rescaling we may assume that the length of $K$ is 1 . It has been observed by Eric Rawdon and Jonathan Simon (unpublished) that given any smooth knot $K$, there is a number $N$ such that the statement is true for all $n>N$. Define an $\epsilon$-chain of length $n$ to be a sequence of points $\left(x_{0}, \ldots, x_{n-1}\right)$ on $K$, lying successively along the positive orientation of $K$, such that $\left\|x_{i}-x_{i-1}\right\|=\epsilon$ for $i=1, \ldots, n-1$. Choose $N$ large enough so that for any $\epsilon<2 / N$ and any point $x$ on $K$ there are exactly two points on $K$ with distance $\epsilon$ from $x$. Then starting from any $x_{0} \in K$ one may construct an $\epsilon$-chain of length $n+1$ for small $\epsilon$. The sum of lengths of the short $\operatorname{arcs}$ on $K$ between adjacent points in the chain is small when $\epsilon$ is small, and exceeds 1 when $\epsilon>1 / N$. Hence by continuity there must be some $\epsilon$ such that $x_{n}=x_{0}$, and the result follows. The proof fails when $n$ is small.

The question of whether every smooth knot admits an inscribed $n$-gon for all $n$ has remained open for some time and no answer is known. It seems worth while to record a positive solution. Actually, a little more is true. One can find a regular polygon with one vertex at any prescribed point. The proof is very elementary, although it does use the concept of degree of maps between spheres in an essential way. See [1] for some background. Note that the theorem as stated is not true if the smoothness assumption is dropped; however, it is not known whether it is true if one is also allowed to move the base point. See Remark 6 and Conjecture 7 below for more details.

1991 Mathematics Subject Classification. Primary 57M25.

Key words and phrases. Smooth knots, regular $n$-gon.

${ }^{1}$ Partially supported by NSF grant \#DMS 9802558 
Theorem 1. Let $K: S^{1} \rightarrow \mathbb{R}^{3}$ be a smooth knot, and let $x_{0} \in K$ be a fixed base point. Then for any $n$ there is a regular polygon of $n$ edges inscribing $K$, containing $x_{0}$ as a vertex.

Proof. We may assume that $n \geq 3$; the result is trivial otherwise. We use the same notation $K$ to denote the image of the map $K$. Composing with $q: \mathbb{R}^{1} \rightarrow S^{1}$, $q(t)=e^{2 \pi t i}$, we have a universal covering map $k: \mathbb{R}^{1} \rightarrow K \subset \mathbb{R}^{3}$. Without loss of generality we may assume that $x_{0}$ is the origin of $\mathbb{R}^{3}$, and $k(0)=x_{0}$. Let $D(r)$ be a ball of radius $r$ centered at $x_{0}$, chosen so that $D(r) \cap K=\gamma$ is a single arc on $K$. Let $J$ be the interval containing 0 such that $k(J)=\gamma$. Denote by $d\left(t^{\prime}, t\right)$ the distance between two points on $K$ with parameters $t^{\prime}$ and $t$, i.e., $d\left(t^{\prime}, t\right)=\left\|k\left(t^{\prime}\right)-k(t)\right\|$.

Lemma 2. There is a $D(r)$ and a positive number $\epsilon$, such that

(a) $\frac{\partial d\left(t^{\prime}, t\right)}{\partial t}>0$ for $t>t^{\prime}$ in $J$

(b) there exist $0=a_{0}<a_{1}<\cdots<a_{n-1}$ in $J$, such that $d\left(a_{i-1}, a_{i}\right)=\epsilon$ for $i=1, \ldots, n-1 ;$ and

(c) if $d\left(a_{i}, t\right)=\epsilon$ for some $i=1, \ldots, n-2$ and $t \in[0,1)$, then $t=a_{i \pm 1}$.

Proof. (a) Since $K$ is smooth, $k^{\prime}(0) \cdot k^{\prime}(0)>0$, so one can choose $r$ small enough so that $k^{\prime}(s) \cdot k^{\prime}(t)>0$ for all $s, t \in J$. Up to changing of coordinate we may assume that $t^{\prime}=0$. Put $h(t)=d\left(t^{\prime}, t\right)^{2}=d(0, t)^{2}=k(t) \cdot k(t)$. If the result were not true then $h^{\prime}(t)=2 k(t) \cdot k^{\prime}(t)=0$. Now $k(t)=\int_{0}^{t} k^{\prime}(s) d s$, hence

$$
k(t) \cdot k^{\prime}(t)=\int_{0}^{t} k^{\prime}(s) \cdot k^{\prime}(t) d s=0,
$$

which is impossible because $k^{\prime}(s) \cdot k^{\prime}(t)>0$ for all $s, t$ in $J$.

(b) Choose $\epsilon<r / n$. Assume $a_{i}$ has been found for some $i<n-1$. Let $b$ be the right endpoint of $J$. Then $d\left(a_{i}, b\right)>r-d\left(a_{i}, 0\right)>r-i \epsilon>\epsilon$. By (a) $d\left(a_{i}, t\right)$ is increasing for $t>a_{i}$, and we have $d\left(a_{i}, a_{i}\right)=0$. Hence the continuity of $d\left(a_{i}, t\right)$ implies that there is a unique $a_{i+1}$ in $\left(a_{i}, b\right)$ satisfying $d\left(a_{i}, a_{i+1}\right)=\epsilon$.

(c) If $k(t) \in D(r)$ the proof follows from that of (b). If $k(t) \notin D(r)$ one can show that $d\left(a_{i}, t\right)>\epsilon$.

We shall assume below that $\epsilon$ and $a_{i}$ have been chosen as in the lemma. Let a denote the vector $\left(a_{1}, \ldots, a_{n-1}\right)$. Note that $a_{0}=0$ is not a component of this vector.

Let $p$ be a number between $a_{n-1}$ and 1 such that $d(p, 0)<\epsilon$. This is possible because $\lim _{p \rightarrow 1} d(p, 0)=0$. Note that $d(0, t)>d(0, p)$ if $t \in\left(a_{1}, p\right)$. Consider the set

$$
B=\left\{\left(t_{1}, \ldots, t_{n-1}\right) \in \mathbb{R}^{n-1} \mid a_{1} \leq t_{1} \leq \ldots \leq t_{n-1} \leq p\right\} .
$$

For notational convenience, we shall always write $t_{0}=0$ and $t_{n}=1$. Notice that $B$ is an $(n-1)$-dimensional simplex.

Denote by $\partial B$ the boundary of $B$. It consists of $n$ faces given by $E=E_{1}=\{\mathbf{t} \in$ $\left.B \mid t_{1}=a_{1}\right\}, E_{i}=\left\{\mathbf{t} \in B \mid t_{i-1}=t_{i}\right\}$ for $2 \leq i \leq n-1$, and $E_{n}=\left\{\mathbf{t} \in B \mid t_{n-1}=\right.$ $p\}$. Note that $\mathbf{a}=\left(a_{1}, \ldots, a_{n-1}\right)$ is an interior point of $E$.

Consider the line

$$
\Delta=\left\{\mathbf{y} \in \mathbb{R}^{n} \mid y_{1}=\cdots=y_{n}\right\},
$$

called the diagonal of $\mathbb{R}^{n}$. Denote by $P$ the plane

$$
P=\left\{\mathbf{y} \in \mathbb{R}^{n} \mid \sum y_{i}=0\right\} .
$$


Note that $P$ is the orthogonal complement of $\Delta$ when $\Delta$ and $P$ are considered as linear subspaces of $\mathbb{R}^{n}$. Define maps

$$
\begin{aligned}
& \varphi: B \rightarrow \mathbb{R}^{n}, \quad \varphi(\mathbf{t})=\mathbf{y}, \quad y_{i}=d\left(t_{i-1}, t_{i}\right) \\
& \psi_{1}: \mathbb{R}^{n} \rightarrow P, \quad \psi_{1}(\mathbf{y})=\mathbf{z}, \quad z_{i}=y_{i}-c, \quad c=\sum y_{i} / n \\
& \psi_{2}: P-\{\mathbf{0}\} \rightarrow S^{n-2}, \quad \psi_{2}(\mathbf{z})=\mathbf{z} /\|z\|
\end{aligned}
$$

Note that $\psi_{1}$ is the orthogonal projection of $\mathbb{R}^{n}$ to $P$, and $\psi_{2}$ is the standard radial projection.

Now suppose the theorem were not true. Then $\varphi(B)$ is disjoint from $\Delta$, hence the map $f=\psi_{2} \circ \psi_{1} \circ \varphi$ is a continuous map from $B$ to $S^{n-2}$, so its restriction to $\partial B$, denoted by $g: \partial B \rightarrow S^{n-2}$, has degree 0 . In the following we will show that $g$ actually has degree \pm 1 . This contradiction will then complete the proof of Theorem 1.

Lemma 3. Suppose $g(\mathbf{t})=g(\mathbf{a})$ for some $\mathbf{t} \in \partial B$. Then $\mathbf{t}=\mathbf{a}$.

Proof. Put $\mathbf{y}=\varphi(\mathbf{a})$, and $\mathbf{z}=\psi_{1}(\mathbf{y})$. By the choice of $\mathbf{a}$, we have $\mathbf{y}=\left(y_{1}, \ldots, y_{n}\right)=$ $(\epsilon, \epsilon, \ldots, \epsilon, \delta)$, where $\delta=d\left(0, a_{n-1}\right)>d\left(0, a_{1}\right)=\epsilon$ because $d(0, t)$ is increasing on $\left[0, a_{n-1}\right]$. Let $c=((n-1) \epsilon+\delta) / n$ be the average of the coordinates of $\mathbf{y}$. Then $\mathbf{z}=(\epsilon-c, \ldots, \epsilon-c, \delta-c)$. Thus $\mathbf{w}=g(\mathbf{a})$, the unit vector in the direction of $\mathbf{z}$, also has the property that $w_{1}=\ldots=w_{n-1}$, and $w_{1}<w_{n}$.

Put $\mathbf{y}^{\prime}=\varphi(\mathbf{t})$, and $\mathbf{w}^{\prime}=g(\mathbf{t})$. Since $\mathbf{w}^{\prime}=g(\mathbf{t})=g(\mathbf{a})=\mathbf{w}$, we have $w_{1}^{\prime}=w_{i}^{\prime}$ for $i<n$, and $w_{1}^{\prime}<w_{n}^{\prime}$. This implies that $y_{1}^{\prime}=y_{i}^{\prime}$ for $i<n$ and $y_{1}^{\prime}<y_{n}^{\prime}$. On the other hand, if $\mathbf{t} \in E_{i} \subset \partial B$ for $1<i<n$ then $t_{i}=t_{i-1}$, so $y_{i}^{\prime}=d\left(t_{i-1}, t_{i}\right)=0$, which is a contradiction because $t_{1}$ is always positive and hence $y_{1}^{\prime}=d\left(t_{1}, 0\right)>0$. Also, if $i=n$, then $t_{n}=p$, so $y_{n}^{\prime}=d(0, p) \leq d\left(0, t_{1}\right)=y_{1}^{\prime}$ because $t_{1} \in\left[a_{1}, p\right]$, which again contradicts the fact that $y_{1}^{\prime}<y_{n}^{\prime}$.

Therefore we must have $\mathbf{t} \in E$. By definition we have $t_{1}=a_{1}$, so $y_{1}^{\prime}=d\left(t_{0}, t_{1}\right)=$ $\epsilon=y_{1}$. The equalities $w_{1}^{\prime}=\ldots=w_{n-1}^{\prime}$ now imply that $y_{i}^{\prime}=d\left(t_{i-1}, t_{i}\right)=y_{1}^{\prime}=\epsilon$ for $i \leq n-1$. By Lemma 2(c), this implies that $t_{i}=a_{i}$, and the result follows.

Lemma 4. The point $\mathbf{a} \in \partial B$ is a regular point of $g$.

Proof. As usual, denote by $g_{*}: T_{\mathbf{a}}(E) \rightarrow T_{g(\mathbf{a})}\left(S^{n-2}\right)$ the map induced by $g$ on the tangent space of $E$ at $\mathbf{a}$. We need to show that the kernel of $g_{*}$ is trivial. Put $\psi=\psi_{2} \circ \psi_{1}$. Then $g_{*}^{-1}(0)=\varphi_{*}^{-1} \circ \psi_{*}^{-1}(0)$. Since $\psi$ is a linear map, $\psi_{*}=\psi$. One can verify that $\psi_{*}^{-1}(0)=\psi^{-1}(0)$ is the linear space $L$ spanned by $\varphi(\mathbf{a})$ and $\mathbf{e}=(1, \ldots, 1)$. To prove the lemma, we need only show that $\mathbb{R}^{n}$ is spanned by $L$ and $\operatorname{Im}\left(\varphi_{*}\right)$, the image of $\varphi_{*}$.

From the definition of $\varphi$, one can see that $\varphi_{*}$ is defined by the following matrix, which has $n$ rows and $n-1$ columns.

$$
\left(\begin{array}{ccccc}
c_{11} & 0 & 0 & \cdots & 0 \\
c_{21} & c_{22} & 0 & \cdots & 0 \\
0 & c_{32} & c_{33} & \cdots & 0 \\
\vdots & \vdots & \vdots & \cdots & \vdots \\
0 & 0 & 0 & \cdots & c_{n-1, n-1} \\
0 & 0 & 0 & \cdots & c_{n, n-1}
\end{array}\right)
$$


The coefficient $c_{i j}$ is the partial derivative of $\varphi_{i}$ with respect to $t_{j}$ at $\mathbf{a}$. The calculation follows from the fact that $\varphi_{i}(\mathbf{t})=d\left(t_{i-1}, t_{i}\right)$ is independent of $t_{j}$ with $j \neq i, i-1$. Recall from the definition of $\mathbf{a}$ that $c_{i i}$ is positive for all $i$.

Since the boundary face $E$ of $B$ is on the plane $t_{1}=a$ in $\mathbb{R}^{n-1}$, the tangent space $T_{\mathbf{a}}(E)$ is spanned by the last $n-2$ vectors of the standard basis of $\mathbb{R}^{n-1}$. Hence $\operatorname{Im}\left(\varphi_{*}\right)$ is generated by the last $n-2$ columns of the above matrix. It follows that $L+\operatorname{Im}\left(\varphi_{*}\right)$ is spanned by the columns of the matrix

$$
M=\left(\begin{array}{cccccc}
\epsilon & 1 & 0 & 0 & \cdots & 0 \\
\epsilon & 1 & c_{22} & 0 & \cdots & 0 \\
\epsilon & 1 & c_{32} & c_{33} & \cdots & 0 \\
\vdots & \vdots & \vdots & \vdots & \vdots & \vdots \\
\epsilon & 1 & 0 & 0 & \cdots & c_{n-1, n-1} \\
\delta & 1 & 0 & 0 & \cdots & c_{n, n-1}
\end{array}\right)
$$

where $\delta=d\left(0, t_{n-1}\right)>\epsilon$ by Lemma 2(a). Subtracting $\epsilon$ times of the second column from the first column and then expanding along the first column, we have $\operatorname{det} M=(-1)^{n+1}(\delta-\epsilon) c_{22} \cdots c_{n-1, n-1} \neq 0$. Hence $\varphi$ is transverse to $P$, and the result follows.

Suppose $h: M \rightarrow N$ is a smooth map between closed oriented smooth manifolds of the same dimension. If $y \in N$ is a regular value of $h$, then the degree of $h$ equals the number of points in $h^{-1}(y)$ at which $h$ is orientation preserving, subtracted by the number of points in $h^{-1}(y)$ at which $h$ is orientation reversing. See [1]. Using smooth approximation, we see that the above is true even if $h$ is only smooth in a neighborhood of $h^{-1}(y)$.

The sphere $\partial B$ is only piecewise smooth as a submanifold of $\mathbb{R}^{n-1}$, but we can compose with a piecewise smooth map $\rho: S^{n-2} \rightarrow \partial B$ to obtain a map $h=g \circ \rho$ : $S^{n-2} \rightarrow S^{n-2}$ which is smooth in a neighborhood of $h^{-1}(g(\mathbf{a}))$. Lemmas 3 and 4 show that $g(\mathbf{a})$ is a regular value of $h$, and $h^{-1}(g(\mathbf{a}))$ has only a single point. Therefore we have $\operatorname{deg}(g)=\operatorname{deg}(h)= \pm 1$. As shown in the paragraph before Lemma 3, this contradicts the facts that $g$ is the restriction of $f: B \rightarrow S^{n-2}$ to $\partial B$, completing the proof of Theorem 1 .

The assumptions in Theorem 1 can be weakened. We have the following generalization.

Theorem 5. Suppose $K: S^{1} \rightarrow \mathbb{R}^{m}$ is a continuous map. Let $x_{0} \in S^{1}$ be a point such that (i) $K$ has nonzero continuous derivative in a neighborhood of $x_{0}$, and (ii) $K(x) \neq K\left(x_{0}\right)$ for all $x \neq x_{0}$. Then for any $n$ there is a regular polygon of $n$ edges inscribing $K$, containing $x_{0}$ as a vertex.

Proof. Note that (i) and (ii) imply that $m \geq 2$. By the compactness of $S^{1}$ one can show that there is a neighborhood $V$ of $x_{0}$ such that (ii) holds when $x_{0}$ is replaced by any $y \in V$. Thus using (i) and (ii) we can still find a ball $D(r)$ of radius $r$ centered at $x_{0}$ such that $D(r) \cap K$ is a single arc. In the proof of Theorem 1 we used smoothness of $K$ in the proofs of Lemmas 2 and 4; however, in either case we only used the fact that $K$ has continuous nonzero derivative in a small neighborhood of $x_{0}$. The rest of the proof of Theorem 1 applies verbatim. 
Remark 6. Condition (i) in Theorem 5 cannot be removed. An easy example is given by a triangle $K$ with two equilateral edges joined at $x_{0}$ at an angle less than $\pi / 3$, in which case there is no regular 3-gon inscribing $K$ with $x_{0}$ as a vertex. However, it is not known if the result would still be true for non smooth knot if it is not required that $x_{0}$ be a vertex of the polygon.

Condition (ii) in Theorem 5 cannot be removed either. The projection $p: \mathbb{R}^{2} \rightarrow$ $\mathbb{R}^{1}$ induces a smooth map $K: S^{1} \rightarrow \mathbb{R}^{1} \subset \mathbb{R}^{3}$, which does not have a regular 3-gon. One can also find a figure 8 curve on the plane with a single double point at $x_{0}$, which is not inscribed by a regular 3-gon with $x_{0}$ as one of its vertex.

Conjecture 7. Any (non smooth) knot $K: S^{1} \hookrightarrow \mathbb{R}^{3}$ is inscribed by a regular $n$-gon for any $n$.

One might attempt to approach the knot $K$ with a sequence of smooth maps $K_{i}$. Let $G_{i}$ be a regular $n$-gon inscribing $K_{i}$. The limit of a convergent subsequence of $G_{i}$ is then an $n$-gon $G$ of equal edge length inscribing $K$. The only problem here is that $G$ might be degenerate in the sense that all of its vertices are at the same point of $K$.

\section{REFERENCES}

[1] V. Guillemin and A. Pollack, Differential Topology, Prentice-Hall, 1974.

[2] V. Klee and S. Wagon, Old and new unsolved problems in plane geometry and number theory, The Dolciani Mathematical Expositions, vol. 11, Mathematical Association of America, Washington, DC, 1991.

[3] E. Kronheimer and P. Kronheimer, The tripos problem, J. London Math. Soc 24 (1981), $182-192$.

[4] Mark Meyerson, Equilateral triangles and continuous curves, Fund. Math. 110 (1980), $1-9$.

[5] M. Nielsen and S. Wright, Rectangles inscribed in symmetric continua, Geom. Dedicata 56 (1995), 285-297.

[6] Walter Stromquist, Inscribed squares and square-like quadrilaterals in closed curves, Mathematika 36 (1989), 187-197.

Department of Mathematics, University of Iowa, Iowa City, IA 52242

E-mail address: wu@math.uiowa.edu 